\title{
Identification of putative natriuretic hormones isolated from human urine
}

\author{
Herbert J. Kramer* \\ Center of Internal Medicine, Rheinische-Friedrich-Wilhelms-University, Bonn, Germany,
}

This brief review describes some representative methodological approaches to the isolation of putative endogenous inhibitors of epithelial sodium transport - i.e., as ouabain-like factors (OLF) that inhibit the sodium transport enzyme Na-K-ATPase or inhibit the epithelial sodium channel (ENaC). Gel chromatography and reverse-phase (RP)-high performance liquid chromatography (HPLC) of lyophilized and reconstituted $24 \mathrm{~h}$-urine from salt-loaded healthy humans led to two active fractions, a hydrophilic OLF-1 and a lipophilic OLF-2, whose mass (Ms)-spectroscopic data indicate a $M_{r}$ of 391 (1, 2). Further identification was attempted by Ms-, infrared (IR)-, ultraviolet (UV)-, and ${ }^{1} \mathrm{H}-\mathrm{NMR}$-spectroscopy. OLF-1 and OLF-2 may be closely related if not identical to (di)ascorbic acid or its salts such as vanadium $(\mathrm{V})-\mathrm{V}^{\mathrm{N}}$-diascorbate with $\mathrm{M}_{\mathrm{r}} 403$ (3) and $\mathrm{V}^{\mathrm{I}}$-diascorbate. OLF-1 and

OPEN ACCESS

Edited by:

Harvey C. Gonick,

University of California Berkeley, USA

Reviewed by:

Harvey C. Gonick,

University of California Berkeley, USA

Vardaman M. Buckalew,

Wake Forest School of Medicine,

USA

*Correspondence:

Herbert J. Kramer

Center of Internal Medicine,

Rheinische-Friedrich-WilhelmsUniversity, Sigmund-Freud-Strasse

25, Bonn D-53127, Germany

hkramer@uni-bonn.de

Specialty section:

This article was submitted to Neuroendocrine Science, a section of the journal Frontiers in Endocrinology

Received: 16 January 2015 Accepted: 14 April 2015

Published: 20 May 2015

Citation:

Kramer HJ (2015) Identification of putative natriuretic hormones isolated from human urine. Front. Endocrinol. 6:66. doi: 10.3389/fendo.2015.00066
$\mathrm{V}^{\mathrm{v}}$-diascorbate are about 10-fold stronger inhibitors of Na-K-ATPase than OLF-2 and $\mathrm{V}^{\mathrm{V}}$-diascorbate, respectively. In conscious rats, i.v. infusion of OLF-1 and OLF-2 resulted in a strong natriuresis. In a similar study, Cain et al. (4) isolated a sodium transport inhibitor from the urine of uremic patients by gel chromatography and RP-HPLC. In uremic rats, a natriuretic response to the injection of the active material was found. Xanthurenic acid 8-O- $\beta$-D-glucoside $\left(M_{r} 368\right)$ and xanthurenic acid 8-O-sulfate $\left(M_{r} 284\right)$ were identified as endogenous inhibitors of sodium transport acting, e.g., by ENaC blockade. No definite relation to blood pressure, body fluid volume, or sodium balance has been reported for any of these above factors, and further studies to identify the natriuretic and/or ouabain-like compound(s) or hormone(s) will be needed.

Keywords: sodium transport, natriuretic hormone, human urine, endogenous inhibitors, epithelial sodium transport

\section{Introduction - Background}

With this brief review, some methological aspects of the isolation of putative endogenous membrane transport inhibitor(s) and natriuretic factor(s) will be described, and the results compared with those of similar attempts by other groups of investigators.

In 1969 (5), and in more detail in 1974 (6) and 1977 (7), we demonstrated for the first time that acute extracellular fluid volume (ECFV)-expansion in rats may release a natriuretic factor or "hormone," which was postulated to act through inhibition of the sodium pump. Thus, ECFV-expansion was accompanied by a decrease in Na-K-ATPase in the renal cortex and the appearance of an inhibitor of Na-K-ATPase in the serum of these rats, respectively. Using gel chromatography, this inhibitory activity was also detected in the post-salt fraction of serum from ECFV-expanded dogs and in the serum and urine of salt-loaded humans. Besides the in vitro-assay of the inhibitory activity, we also demonstrated the inhibitory effect of this serum fraction on epithelial sodium transport, i.e., on 
short-circuit current (SCC) and potential difference (PD) in the isolated frog skin (8). This fraction of serum or urine was also found to cause natriuresis in a rat bioassay. We concluded that a natriuretic factor emerges in the circulation with excessive salt load whose mechanism of action was to modulate the Na-K-ATPase enzyme in the vasculature as well as in the renal tubule.

Attempts to identify this humoral inhibitor(s), however, remained unsuccessful despite the use of extensive methodologic approaches. Besides an endogenous ouabain (9), several bufodienolides (10) have been identified using the methods described in this paper of which two compounds with $\mathrm{M}_{\mathrm{r}}$ of around 400 daltons will be considered in the present mini-review, namely vanadium (V)-diascorbate(s) and derivatives of xanthurenic acid (4). The compounds were assumed to be present in the circulation, and therefore may be excreted in the urine.

\section{Ouabain-Like Factor(s) as Endogenous Sodium Transport Inhibitors}

As source for isolation and identification of a putative natriuretic hormone and/or endogenous epithelial sodium transport enzyme inhibitor, we pooled large quantities (50-100 L) of urine from salt-loaded healthy humans, which was lyophilized to dryness and reconstituted with $0.01 \mathrm{M}$ acetic acid, and then subjected to gel chromatography using Sephadex G-25 and Sephadex G-10 columns.

\section{Ouabain-Like Factors and Vanadium-Diascorbic Acid: Effects on Na-K-ATPase}

To detect the serum and urine fractions with the active compound(s), we employed an in vitro-assay of Na-K-ATPase using a purified commercially available hog cerebral enzyme preparation. We also used this Na-K-ATPase membrane fraction as marker to follow-up activity with purification steps during the subsequent chromatographic steps. To detect the potential natriuretic activity, all fractions were screened for their natriuretic effect using a bioassay in conscious rats (11).

The transport enzyme Na-K-ATPase inhibitory and natriuretic activity(ies) eluted from the Sephadex G-25 column in a post-salt fraction. When this fraction was then subjected to gel chromatography on Sephadex G-10, a strongly active enzyme inhibitory material eluted in a late fraction (1). This late fraction also showed a significant natriuretic action (11). This enzyme inhibitory and natriuretic fraction was subjected to high performance liquid chromatography (HPLC), and subsequently to thin layer chromatography (TLC). Characterization of the active material was attempted by mass $\left(\mathrm{M}_{\mathrm{r}}\right)$-, nuclear magnetic resonance $\left({ }^{1} \mathrm{H}-\mathrm{NMR}\right)$-, infrared (IR)spectroscopy (1), and ultraviolet (UV)-fluorescence/absorbance. The natriuretic activity was also studied by bioassay to identify the active compounds after gel filtration, reverse phase (RP)-HPLC, and amino acid analysis for its potential peptidic character (11).

Reverse-phase HPLC of this highly active late fraction from Sephadex G-10 resulted in two subfractions with significant Na-KATPase enzyme inhibition. They were named ouabain-like factors (OLF); one eluted in the water phase as the more polar hydrophilic OLF-1; the second eluted in a later phase at $20 \%$ acetonitrile as the more apolar lipophilic OLF-2. These fractions also produced a significant natriuresis (see below).

\section{Analysis of Chemical Structure}

Both compounds showed signals for hydroxyl and carboxyl groups as well as criteria for esters or lactones (a precursor of ascorbic acid in plants and animals is L-gulono-y-lactone, and 2,3-diketogulonic acid is an oxidation product of ascorbic acid). No signals for aromatic, aliphatic, heterocyclic, or steroid structures were found. Whereas the IR-spectrum of OLF-1 is different from that of OLF-2 (1), UV-, $\mathrm{M}_{\mathrm{r}}{ }^{-}$, and ${ }^{1} \mathrm{H}-\mathrm{NMR}$-criteria were similar and fluorescence of both compounds when separated by TLC required the presence of a dicarboxylic acid-like conformation; dicarboxylic acid [see also Ref. (11): Asp, Glu as carboxylic acids] is an organic compound containing two carboxyl functional groups (-COOH). IR- and ${ }^{1} \mathrm{H}$ NMR spectra of OLF-1 and OLF-2 suggest a chemical structure resembling a sugar or sugar derivative. However, sugars are not fluorescent as are the OLF recovered from TLC. Therefore, these data suggest the unknown compounds to be identical with ascorbic acid or its salts such as $\mathrm{V}^{\mathrm{v}}$-diascorbate and $\mathrm{V}^{\mathrm{IV}}$-diascorbate, respectively, with $M_{r} 403$ (3). The superscript roman numbers indicate the oxidative state of vanadium $(\mathrm{V})$ : $\mathrm{V}^{\mathrm{lV}}$ oxide $\left(\mathrm{V}_{2} \mathrm{O}_{5}\right)$, the most stable oxygen combination, and $\mathrm{V}^{\mathrm{lV}}$ oxide $\left(\mathrm{VO}_{2}\right)$ represent two of the four oxygen states of vanadium. V-diascorbates elute from the RP-HPLC column at similar elution times and acetonitrile gradients as the hydrophilic and lipophilic OLF-1 and OLF-2, respectively. $\mathrm{V}^{\mathrm{IV}}$ diascorbate also showed the same UV-maximum as we found for OLF. Thus, ascorbic acid seems to be an important cornerstone of the structure of the yet unknown humoral ATPase inhibitor.

It is noteworthy that the water solubility of the individual ascorbic acid salts of metals varies remarkably, and it may be assumed that $\mathrm{V}^{\mathrm{v}}$ - and $\mathrm{V}^{\mathrm{lV}}$ diascorbates with their different water solubility elute from the RP-HPLC column at similar elution times as the OLF-1 and OLF-2, respectively. V-diascorbates also show the same UV-maximum as the OLF and are strong candidates for the urinary hydrophilic OLF-1 and lipophilic OLF-2, respectively.

\section{Effects on Enzyme Kinetics}

These active subfractions, containing OLF-1 and OLF-2, were further purified by two-dimensional preparative TLC to single compounds, whose mass spectroscopic (MS) data suggested a $\mathrm{M}_{\mathrm{r}}$ of around 400. Actually, OLF-2, which dose-dependently inhibited Na-K-ATPase, was found to have a $M_{r}$ of 391 (1). With respect to the effects of OLF- 1 and OLF- 2 and of $\mathrm{V}^{\mathrm{v}}$ - and $\mathrm{V}^{\mathrm{IV}}$-diascorbates on $\mathrm{Na}-\mathrm{K}-\mathrm{ATPase}$ enzyme activity and kinetics, in vitro studies showed that OLF-1 and OLF-2 inhibited the enzyme in its E2 configuration. In analogy to the polar OLF-1, which revealed an approximately 10 -fold stronger enzyme inhibition $\left(\mathrm{IC}_{50} 1.5 \times 10^{-5} \mathrm{M}\right)$ than the apolar OLF-2 $\left(\mathrm{IC}_{50} 1.5 \times 10^{-4} \mathrm{M}\right)$, we found that $\mathrm{V}^{\mathrm{v}}$-diascorbate $\left(\mathrm{IC}_{50} 2 \times 10^{-6} \mathrm{M}\right)$ is a significantly stronger inhibitor of Na-KATPase than $\mathrm{V}^{\mathrm{IV}}$-diascorbate $\left(\mathrm{IC}_{50}\right.$ of $\left.9 \times 10^{-5} \mathrm{M}\right)(3,5,12)$. In this context, I should mention that we found previously that certain trace metals are strong inhibitors of this enzyme (13).

\section{Renal and Vascular Mechanisms of Action of OLF}

Regarding the potential mechanism of the physiological and pathological effects of OLF-1 and OLF-2 on vascular smooth muscle cells (VSMCs) and inner medullary collecting duct cells (IMCD cells), we found in an in vitro-assay that OLF-1 and OLF-2 enhanced 
VSMC contractility by increasing intracellular $\mathrm{Ca}^{2+}$ similar to the effect of ouabain $(14,15)$. Similar effects were found with OLF-1 and OLF- 2 on intracellular $\mathrm{Ca}^{2+}$ in IMCD cells, suggesting inhibition of tubular Na-reabsorption and thus regulating renal excretion, i.e., to enhance Na-excretion (16).

\section{Ouabain-Like Factors and V-Diascorbates: Natriuretic Effects}

For demonstration of the natriuretic activity, we used a bioassay in conscious rats (12). As mentioned above, in our assay system, the post-salt fraction IV from Sephadex G-25 was applied to Sephadex-G-10 and resulted in a late fraction, which was applied to RP-HPLC. When administered i.v., OLF-1 resulted in an immediate, eightfold rise in natriuresis from approximately 1 to $8 \mu \mathrm{Eq} / \mathrm{min} / \mathrm{mg}$, whereas the apolar OLF-2 caused a natriuresis of slower onset reaching its maximum after $60 \mathrm{~min}$ and lasting for more than $180 \mathrm{~min}$. This was confirmed also by injection of the active fractions obtained by quantitative TLC.

\section{Natriuretic Factor Unrelated to OLF}

Finally, I should mention that we described previously a natriuretic compound, which we suggested to be a peptide. Thus, when the pooled post-salt natriuretic urine fraction obtained by gel chromatography (see above) was subjected to repetitive RP-HPLC, a late eluting fraction showed strong natriuretic activity in the bioassay and was associated with a fluorescence peak when treated with o-phthaldialdehyde as a marker for primary amines (11). Amino acid analysis before and after total acid hydrolysis suggested a peptide tentatively containing the amino acids (AA) Asp, Glu, Gly, Phe, and Ser $(1,11)$. The natriuretic activity was lost after incubation with chymotrypsin, which splits bonds with aromatic AA (2). We found, in addition, that several synthetic (mono-) peptides of di- and tri-AA are significantly natriuretic when injected i.v. (unpublished data).

\section{Xanthurenic Acid 8-0- $\beta$-D-Glucoside and Xanthurenic Acid 8-0-Sulfate as Endogenous Sodium Transport Inhibitors}

Cain et al. (4) followed a protocol very similar to that of Kramer et al. for isolation of the natriuretic activity except that they used the urine of uremic patients as source of the inhibitor and a bioassay in (conscious?) uremic rats. As marker for the active material, Cain et al. used changes of the SCC of the isolated frog skin - as we described in 1977 (8) - for monitoring transepithelial sodium transport inhibitory activity. For monitoring its natriuretic effect, the above mentioned bioassay in uremic rats was used. A direct in vitro-assay for inhibition of the Na-K-ATPase enzyme by the natriuretic factor or "hormone" was not employed. The authors rather speculate that the natriuretic hormone may act via other sodium pumps in the kidney, e.g., the epithelial sodium channel $(\mathrm{ENaC})$ in the distal tubule.

\section{Xanthurenic Acid Derivatives: Effects on Epithelial Sodium Transport}

Epithelial sodium transport was measured as changes of SCC and PD in the isolated frog skin. For isolation and identification of the transport inhibitor, one gel chromatographic step and three consecutive HPLC steps were applied. Final identification was achieved by mass (Ms)-, IR-, UV-, and NMR-spectroscopy. Purification of the activity was estimated from UV peak with a characteristic spectrum at $338 \mathrm{~nm}$. Xanthurenic acid 8-O- $\beta-\mathrm{D}$ glucoside $\left(\mathrm{M}_{\mathrm{r}} 368\right)$ and xanthurenic acid 8-O-sulfate $\left(\mathrm{M}_{\mathrm{r}} 284\right)$ were identified as the endogenous sodium transport $(\mathrm{ENaC}$, Na-K-ATPase) inhibitors.

\section{Xanthurenic Acid Derivatives: Natriuretic Effects}

The material (M 368) obtained from two HPLC runs was tested for natriuretic effect in their uremic rat bioassay. Urinary sodium excretion rose immediately and reached its maximum approximately $40 \mathrm{~min}$ after intra-arterial infusion (5). Urinary volume increased slightly and then decreased to below baseline, i.e., a decrease in urine volume with a rise in urinary osmolality.

A pathophysiological role of xanthurenic acid, a tryptophane derivative, is difficult to envisage as this uremic toxin may inhibit transmembranous sodium transport independent of a potential role as specific circulating natriuretic or sodium transport inhibiting "hormone." Thus, although Bricker et al. showed that the natriuretic action of the isolated inhibitor paralleled the changes in renal function, as an alternative explanation, it may be reasonable to assume that with the progressive decrease in renal function and the accumulation of toxic metabolites, the rise in fractional sodium excretion may parallel the urinary concentration of the xanthurenic derivatives.

\section{Summary}

Although there is no doubt that an as yet unidentified natriuretic compound can be isolated from human urine by gel filtration and RP-HPLC, whose activity changes in parallel with salt (sodium chloride) intake, i.e., it correlates with salt-balance (low or high salt intake). Therefore, the activity may be related to an as yet unidentified "natriuretic hormone" that is assumed to play a crucial role in the fine-tuning of renal tubular sodium handling and may thus be involved in the long-term body fluid and blood pressure regulation.

We found two Na-K-ATPase inhibitors, the hydrophilic OLF-1 and the lipophilic OLF-2 (1). The hydrophilic form was more potent than the lipophilic one. The lipophilic compound was moderately natriuretic but strong ATPase inhibitor. Both compounds showed UV fluorescence/absorbance of lower intensity in the hydrophilic (hydrated) form. Both enzyme inhibitors showed UV-absorbance, which requires the presence of a dicarboxylic acid-like arrangement (1). In addition, from our data the unknown compound(s) most likely fulfill(s) the criteria for lactones.

Unfortunately, for none of the three classes of endogenous sodium transport inhibitors, a physiologic or pathophysiologic role was demonstrated, i.e., no correlation to body fluid and sodium balance or blood pressure was documented. Thus, further studies are required to confirm the structures of the various endogenous factors; final identification of their physiological and pathophysiological significance must await urgent results of additional welldesigned studies. 


\section{References}

1. Kramer HJ, Krampitz G, Bäcker A, Michel H, Krampitz G Jr. Meyer-Lehnert H. Endogenous sodium pump inhibitors in human urine. Further identification of inhibitors of Na-K-ATPase. Am J Hypertens (1995) 8:753-60. doi:10.1016/0895-7061(95)00125-9

2. Kramer HJ, Meyer-Lehnert H, Michel H, Predel HG. Endogenous natriuretic and ouabain-like factors. Their roles in body fluid and blood pressure regulation. Am J Hypertens (1991) 4:81-9.

3. Kramer HJ, Krampitz G, Bäcker A, Meyer-Lehnert H. Ouabain-like factors in human urine: identification of a Na-K-ATPase inhibitor as vanadium-diascorbate adduct. Clin Exp Hypertens (1998) 20(5\&6):557-71. doi:10.3109/10641969809053234

4. Cain CD, Schroeder FC, Shankel SW, Mitchnick M, Schmertzler Bricker NS. Identification of xanthurenic acid 8-O-ß-D-glucoside and xanthurenic acid 8-O-sulfate as human natriuretic hormones. Proc Natl Acad Sci U S A (2007) 104:17873-8. doi:10.1073/pnas.0705553104

5. Kramer HJ, Gonick HC, Paul W, Lu E. Third factor: inhibitor of Na-K-ATPase? Abstracts free comm. IVth International Congress of Nephrology. Stockholm, Sweden (1969). p. 373

6. Kramer HJ, Gonick HC. Effects of extracellular volume expansion on renal Na-KATPase and cell metabolism. Nephron (1974) 12:281-96. doi:10.1159/000180341

7. Gonick HC, Kramer HJ, Paul W, Lu E. Circulating inhibitor of sodium-potassium-activated triphosphatase after expansion of extracellular fluid volume in rats. Clin Sci Mol Med (1977) 53:320-34.

8. Kramer HJ, Bäcker A, Krück F. Antinatriferic activity in human plasma following acute and chronic salt-loading. Kidney Int (1977) 12:214-22. doi:10.1038/ ki.1977.103

9. Hamlyn JM, Blaustein MP. Salt sensitivity, endogenous ouabain and hypertension. Curr Opin Nephrol Hypertens (2013) 22:51-8. doi:10.1097/ MNH.0b013e32835b36ec

10. Bagrov AY, Shapiro JI, Federova OV. Endogenous cardiotonic steroids: physiology, pharmacology, and novel therapeutic targets. Pharmacol Rev (2009) 61:9-38. doi:10.1124/pr.108.000711
11. Kramer HJ, Heppe M, Weiler E, Bäcker A, Liddiard C, Klingmüller D. Further characterization of the endogenous natriuretic and digoxin-like immunoreacting activities in human urine. Effects of changes in sodium intake. Ren Physiol (1985) 8:80-9.

12. Kramer HJ, Krampitz G, Bäcker A, Meyer-Lehnert H. Vanadium diascorbates are strong candidates for endogenous ouabain-like factors in human urine: effects on Na-K-ATPase enzyme kinetics. Biochem Biophys Res Commun (1995) 213:289-94. doi:10.1006/bbrc.1995.2128

13. Kramer HJ, Gonick HC, Lu E. In vitro-inhibition of Na-K-ATPase by trace metals. Relation to cardiovascular and renal damage. Nephron (1986) 44:329-36. doi: $10.1159 / 000184015$

14. Meyer-Lehnert H, Wanning C, Michel H, Bäcker A, Kramer HJ. Cellular mechanisms of action of a ouabain-like factor in vascular smooth muscle cells. JCardiovasc Pharmacol (1993) 22(Suppl 2):S16-9. doi:10.1097/00005344-199312000-00035

15. Meyer-Lehnert H, Bäcker A, Kramer HJ. Inhibitors of Na-K-ATPase in human urine: effects of ouabain-like factors and of vanadium-diascorbate on calcium mobilization in rat vascular smooth muscle cells. Comparison with the effects of ouabain, angiotensin II, and arginine-vasopressin. Am J Hypertens (2000) 13:364-9. doi:10.1016/S0895-7061(99)00197-1

16. Kramer HJ, Bäcker A, Meyer-Lehnert H. Effects of urinary ouabain-like factor (OLF) and vanadium-diascorbate on calcium mobilization in inner medullary collecting duct (IMCD) cells. Am J Hypertens (1998) 11:1208-13. doi:10.1016/ S0895-7061(98)00134-4

Conflict of Interest Statement: The author declares that the research was conducted in the absence of any commercial or financial relationships that could be construed as a potential conflict of interest.

Copyright (C) 2015 Kramer. This is an open-access article distributed under the terms of the Creative Commons Attribution License (CC BY). The use, distribution or reproduction in other forums is permitted, provided the original author(s) or licensor are credited and that the original publication in this journal is cited, in accordance with accepted academic practice. No use, distribution or reproduction is permitted which does not comply with these terms. 